\title{
Comparison of Three Formulations for Eddy-Current and Skin Effect Problems
}

\author{
R. Jafari-Shapoorabadi, Student Member, IEEE, A. Konrad, Fellow, IEEE, and A. N. Sinclair, Member, IEEE
}

\begin{abstract}
Three finite-element formulations based on different definitions of current density are compared. Formulations I and II are based on incomplete equations for total and source current densities, respectively. Formulation III is based on a complete equation for source current density. To validate the third formulation, a one-dimensional test problem is solved analytically for the magnetic field intensity. The formulations are applied to a nondestructive testing example and a three-phase bus-bar example. Results show that errors due to the use of incomplete equations for current densities increase with frequency and conductor dimensions.
\end{abstract}

Index Terms -Eddy currents, finite elements, skin effect.

\section{INTRODUCTION}

$\mathbf{T}$ HE PHENOMENA of eddy current and skin effect occur in most electrical power devices and in nondestructive testing (NDT) as well. Their accurate simulation in both transient and steady state regimes is often necessary at the design stage.

Review of the technical literature reveals numerous contributions to the solution of eddy current and skin effect problems in both frequency and time domains [1]-[9]. The two-dimensional (2-D) finite-element method (FEM) is used to solve the diffusion equation for the magnetic vector potential (MVP) in [1]-[6]. In [1] and [2], the assumption is made that the total current density (TCD) in a conductor equals total current divided by conductor cross-sectional area. By contrast, in [3] and [4], the source current density (SCD) in a conductor is assumed to equal the ratio of total current and conductor cross-sectional area. According to electromagnetic theory, the SCD depends not only on total current but also on the time derivative of the integral of the MVP over the conductor cross-sectional area. Ignoring the role of the MVP in the expression for the SCD is tantamount to neglecting skin and proximity effects.

The problem is solved more accurately in [7] and [8] by obtaining both MVP and SCD simultaneously. In [11], [12], an improved version of the finite-element (FE) formulation [8] is applied to the analysis of electromagnetic acoustic transducers

Manuscript received July 2, 2001; revised October 25, 2001. This work was supported by the Natural Sciences and Engineering Research Council (NSERC) of Canada, by ANDEC Manufacturing of Toronto, and by the Ontario Centre for Automotive Materials and Manufacturing.

R. Jafari-Shapoorabadi and A. Konrad are with the Edward S. Rogers Sr. Department of Electrical and Computer Engineering, University of Toronto, Toronto, ON M5S 3G4, Canada (e-mail: jafari@ele.utoronto.ca; konrad@ecf.utoronto.ca).

A. N. Sinclair is with the Department of Mechanical and Industrial Engineering, University of Toronto, Toronto, ON M5S 3G8, Canada (e-mail: sinclair@mie.utoronto.ca).

Publisher Item Identifier S 0018-9464(02)01244-X.
(EMATs). Unlike in the original formulation [8], the MVP is the only unknown in the improved formulation [11]. The formulation in [7] and [8] yields a mixed set of algebraic and differential equations. The improved formulation in [11] yields only a differential equation. As a result, the associated numerical method is more stable for transient analysis involving current sources.

\section{MAGNeTIC FiELD EQUATIONS}

Consider the general geometry of several current-carrying conductors in the vicinity of a region (e.g., some metal), which is also conducting. For 2-D multiconductor analysis, the magnetic field is obtained in terms of the MVP in the region of solution, which contains all sources, from the following equations (see the Appendix):

$$
\begin{gathered}
\operatorname{div} \frac{1}{\mu} \operatorname{grad} A_{z}=-J_{z k} \\
J_{z k}=J_{s k}-\sigma \frac{\partial A_{z}}{\partial t} \\
\iint_{R_{k}} J_{z k} d s=i_{k}(t)
\end{gathered}
$$

where $\mu, \sigma$ and $A_{z}$ are permeability, conductivity and MVP, respectively, and $J_{z k}, J_{s k}, i_{k}(t)$, and $R_{k}$ denote TCD, SCD, total current, and cross-sectional region of the $k$ th conductor, respectively. In source-free conducting regions the MVP must satisfy

$$
\operatorname{div} \frac{1}{\mu} \operatorname{grad} A_{z}-\sigma \frac{\partial A_{z}}{\partial t}=0
$$

\section{A. Earlier Formulation I}

In this formulation the TCD is assumed to be independent of position inside a conductor. Thus, from (3)

$$
J_{z k}=\frac{i_{k}(t)}{S_{k}}
$$

where $i_{k}(t)$ is total current and $S_{k}=\iint_{R_{k}} d s$ is the cross-sectional area of the $k$ th conductor [1], [2]. In this paper, (5) is referred to as the incomplete equation for TCD. By substituting (5) into (1), the differential equation for the source region becomes

$$
\operatorname{div} \frac{1}{\mu} \operatorname{grad} A_{z}=-\frac{i_{k}(t)}{S_{k}} .
$$

The discretized FE equivalents of (4) and (6) are obtained from the following equation by assuming that $\sigma=0$ for source regions and by interpreting $J$ as TCD:

$$
\sigma T \frac{d A}{d t}+\frac{1}{\mu} S A=T J .
$$


Here $A_{n \times 1}, J_{n \times 1}, S_{n \times n}$, and $T_{n \times n}$ are the column matrices of FE node potentials, node current density values, and the usual FE coefficient matrices $S$ and $T$ [5], respectively.

\section{B. Earlier Formulation II}

In general, both $A_{z}$ and $J_{s k}$ are unknown. However, in this formulation, which is still often used, $J_{s k}$ is assumed to be only a function of total current. In [5] and [6], the problem is solved for a given SCD. However, it is not clear how the SCD is determined from total current. In many applications the total currents in the conductors are known. For time domain field analysis, as for static field analysis, the following simple equation for $J_{s k}$ is used [3], [4]:

$$
J_{s k}=\frac{i_{k}(t)}{S_{k}} .
$$

In this paper, (8) is referred to as the incomplete equation for SCD. By substituting (2) into (1), the governing differential equation becomes

$$
\operatorname{div} \frac{1}{\mu} \operatorname{grad} A_{z}-\sigma \frac{\partial A_{z}}{\partial t}=-J_{s k} .
$$

The FE equivalent of (9) is given by [6]

$$
\sigma T \frac{d A}{d t}+\frac{1}{\mu} S A=T J_{s}
$$

where $J_{s, n \times 1}$ is the column matrix of nodal SCD values. In frequency domain (10) becomes

$$
\left(\frac{1}{\mu} S+j \omega \sigma T\right) \bar{A}=T \bar{J}_{s}
$$

where $\bar{A}$ and $\bar{J}_{s}$ are the phasors of $A_{n \times 1}$ and $J_{s, n \times 1}$, respectively.

\section{Improved Formulation III}

By making use of the integral form of Maxwell's second equation, $J_{s k}$ is obtained as follows (see the Appendix):

$$
J_{s k}=\frac{i_{k}(t)}{S_{k}}+\frac{\sigma}{S_{k}} \frac{\partial}{\partial t} \iint_{R_{k}} A_{z} d s .
$$

In this paper, (12) is referred to as the complete equation for SCD. By substituting (12) into (9), the governing equation becomes

$$
\operatorname{div} \frac{1}{\mu} \operatorname{grad} A_{z}-\sigma \frac{\partial A_{z}}{\partial t}+\frac{\sigma}{S_{k}} \frac{\partial}{\partial t} \iint_{R_{k}} A_{z} d s=-\frac{i_{k}(t)}{S_{k}} .
$$

The FE equivalent of (13) is more stable than the FE equation used in [8] and is given by

$$
\frac{1}{\mu} S A+\sigma\left(T-Q P^{-1} Q^{T}\right) \frac{d A}{d t}=Q P^{-1} I(t) .
$$

In (14) the rectangular matrix $Q_{n \times m}$ is exactly as given in [8]. $P_{m \times m}^{-1}$ is a diagonal matrix with diagonal elements given by $p_{k k}=S_{k}, k=1, m$, where $m$ is the total number of conductors with SCD. The SCD column matrix, $J_{s, m \times 1}$, is a function of total current and MVP, and is defined as

$$
J_{s}=P^{-1}\left(I+\sigma Q^{T} \frac{d A}{d t}\right) .
$$

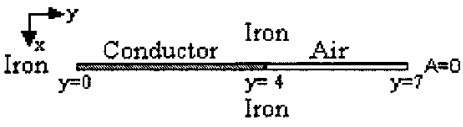

Fig. 1. Thin slot-embedded conductor surrounded by infinitely permeable iron on three sides.

In frequency domain (14) becomes

$$
\left[\frac{1}{\mu} S+j \omega \sigma\left(T-Q P^{-1} Q^{T}\right)\right] \bar{A}=Q P^{-1} \bar{I}
$$

where $\bar{A}$ and $\bar{I}$ are the phasors of $A(x, y, t)$ and $I(t)$, respectively. Note that (7), (10), (11), (14)-(16) are written for just one FE. Usual FE matrix assembly procedures are applied to obtain similar matrix equations which are valid for the entire problem region. Also, it should be noted that only current sources are considered in this paper. With the approach described in [7], the improved formulation may be extended to include voltage sources as well.

\section{VALIDATION}

The test problem shown in Fig. 1 is the classical one-dimensional (1-D) problem of a single, thin, slot-embedded conductor. Geometry and physical properties are identical to those in [9]. The conductor is 4 units high, has conductivity $\sigma=1$, and carries an exponentially decaying total current given by $i(t)=$ $4 e^{-t} u(t)$, where $u(t)$ is the unit step function. Permeability of both conductor and air is taken as $\mu=1$. The governing differential equation in terms of the magnetic field intensity $\vec{H}=H_{x}(y, t) \vec{a}_{x}$ is given by

$$
\frac{\partial^{2} H_{x}}{\partial y^{2}}-\mu \sigma \frac{\partial H_{x}}{\partial t}=0 .
$$

Analytical solution is obtained by separation of variables

$$
\begin{array}{r}
H_{x}(y, t) \\
\quad= \begin{cases}-4 e^{-t} \csc (4) \sin (y), & \text { for } 0 \leq y \leq 4 ; t \geq 0 \\
-4 e^{-t}, & \text { for } 4<y \leq 7 ; t \geq 0 .\end{cases}
\end{array}
$$

Since $J_{z}=-\partial H_{x} / \partial y=-\partial^{2} A_{z} / \partial y^{2}$, one obtains

$$
\begin{array}{r}
J_{z}(y, t) \\
= \begin{cases}4 e^{-t} \csc (4) \cos (y), & \text { for } 0 \leq y \leq 4 ; t \geq 0 \\
0, & \text { for } 4<y \leq 7 ; t \geq 0 .\end{cases}
\end{array}
$$

By integrating $H_{x}=-\partial A_{z} / \partial y$, the MVP is obtained as

$$
\begin{aligned}
& A_{z}(y, t) \\
& = \begin{cases}4 e^{-t} \csc (4) \cos (y)+C_{1}(t), & \text { for } 0 \leq y \leq 4 ; t \geq 0 \\
4 e^{-t} y+C_{2}(t), & \text { for } 4<y \leq 7 ; t \geq 0 .\end{cases}
\end{aligned}
$$

From (2), $J_{s}=J_{z}+\sigma \partial A_{z} / \partial t$, which gives

$$
J_{s}(t)=d C_{1}(t) / d t, \quad \text { for } 0 \leq y \leq 4 ; \quad t \geq 0 .
$$

Note that the TCD, $J_{z}$, is a function of position and time but the $\mathrm{SCD}, J_{s}$, is only a function of time.

The solution for the MVP contains $C_{1}(t)$ and $C_{2}(t)$ and the solution for the SCD includes $d C_{1}(t) / d t$. Thus, the analytical solutions show that $H_{x}$ and $J_{z}$ do not depend on boundary values, but $A_{z}$ and $J_{s}$ do, indicating that the latter are not unique. 


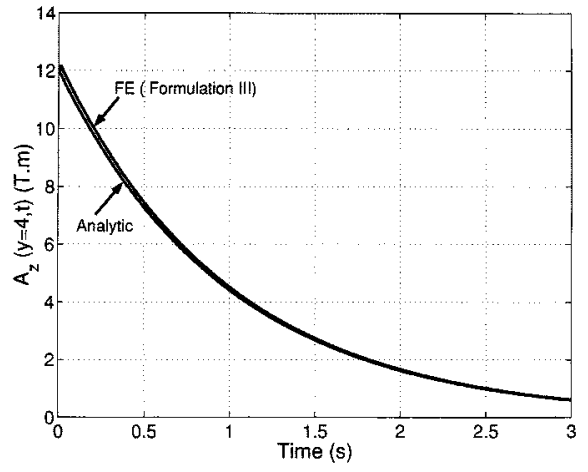

Fig. 2. Comparison of 1-D analytical and 2-D FE results for $A_{z}(y=4, t)$ of the test problem. The FE solution was obtained with $\Delta t=0.01 \mathrm{~s}, 123$ nodes and 192 first-order triangular finite elements.

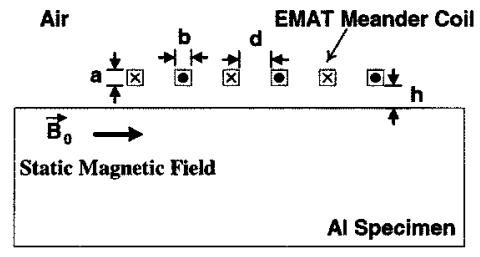

Fig. 3. EMAT meander coil configuration and static magnetic field.

To obtain the MVP, assume that $A_{z}(y=7, t)=0$. With this assumption, $C_{1}(t)$ and $C_{2}(t)$ can be computed. Therefore, $A_{z}(y, t)$ is given by

$$
A_{z}(y, t)=\left\{\begin{array}{l}
4 e^{-t}\{3+[\cos (y)-\cos (4)] \csc (4)\}, \\
4 e^{-t}(y-7), \quad \text { for } 0 \leq y \leq 4 ; t \geq 0 \\
-15 \leq y \leq 7 ; t \geq 0
\end{array}\right.
$$

and $J_{s}(t)=-[12-4 \cot (4)] e^{-t}$ for $0 \leq y \leq 4 ; t \geq 0$. Analytical and FE results for $A_{z}(y=4, t)$ are shown in Fig. 2. The FE grid is defined over the region $0 \leq x \leq 1$ and $0 \leq y \leq$ 7. The average of FE results for $A_{z}(y=4, t)$ at $x=0$ and $x=1$ is displayed in Fig. 2 .

For the above test problem, Formulation II yields a SCD of $J_{s}(t)=e^{-t} u(t)$ while Formulation I gives a TCD of $J_{z}(t)=$ $e^{-t} u(t)$. The discrepancy between these results and the above analytical solution is large.

Note, another analytical solution was obtained and used to validate a computer program based on FE equation (10) for Formulation II. The corresponding test problem is described in [10].

\section{EMAT EXAMPLE}

Fig. 3 shows the 2-D configuration of an electromagnetic acoustic transducer (EMAT) meander coil above an aluminum specimen. The coil consists of a single long wire going back and forth in a series of parallel lines and is used to induce eddy currents in the specimen. The coil in Fig. 3 has six parallel lines of $a$ by $b$ rectangular cross section. Dimensions $h$ and $d$ refer to lift-off and the distance between parallel lines, respectively. The excitation current of the $k$ th conductor is assumed to be $i_{k}(t)=(-1)^{k} I_{0} \cos \left(\omega_{0} t\right)$, where $I_{0}$ is a constant current and $\omega_{0}=2 \pi f_{0}$ is the angular center frequency. Other parameters used are $I_{0}=10 \mathrm{~A}, B_{0}=1 \mathrm{~T}, d=0.5 \mathrm{~mm}$, and $h=1.0 \mathrm{~mm}$.

Table I shows Lorentz force densities $F_{1}, F_{2}, F_{3}$ near the surface of the specimen, directly under meander coil conductors 1 ,
TABLE I

ForCe Density MAgNitudes IN $M N / m^{3}$ InSIDE THE SPECIMEN UNDER THE MEANDER COIL CONDUCTORS. CASE 1: $f_{0}=100 \mathrm{kHz} ; a=0.1 \mathrm{~mm} ; b=0.5$ mm. CASE 2: $f_{0}=500 \mathrm{kHz} ; a=0.1 \mathrm{~mm} ; b=0.5 \mathrm{~mm}$. CASE $3: f_{0}=2 \mathrm{kHz}$; $a=0.1 \mathrm{~mm} ; b=0.5 \mathrm{~mm}$. CASE $4: f_{0}=2 \mathrm{kHz} ; a=0.1 \mathrm{~mm} ; b=1.0 \mathrm{~mm}$

\begin{tabular}{|c|c|c|c|c|c|c|c|c|c|c|c|c|}
\hline & \multicolumn{3}{|c|}{ Case 1} & \multicolumn{3}{|c|}{ Case 2} & \multicolumn{3}{|c|}{ Case 3} & \multicolumn{3}{|c|}{ Case 4} \\
\hline & \multicolumn{3}{|c|}{ Formulation } & \multicolumn{3}{|c|}{ Formulation } & \multicolumn{3}{|c|}{ Formulation } & \multicolumn{3}{|c|}{ Formulation } \\
\hline & I & II & III & I & II & III & I & II & III & 1 & II & III \\
\hline $\mathbf{F}_{1}$ & 8.12 & 5.62 & 8.11 & 18.41 & 4.22 & 18.28 & 23.62 & 1.46 & 23.02 & 26.42 & 1.06 & 24.34 \\
\hline$\overline{F_{2}}$ & 1.05 & 2.17 & 1.01 & 2.69 & 2.27 & 2.38 & 3.88 & 0.81 & 3.00 & 12.84 & 0.85 & 10.11 \\
\hline $\mathrm{F}_{3}$ & 2.90 & 2.13 & 2.91 & 7.71 & 2.40 & 7.70 & 10.51 & 0.89 & 10.18 & 18.91 & 1.02 & 16.66 \\
\hline
\end{tabular}

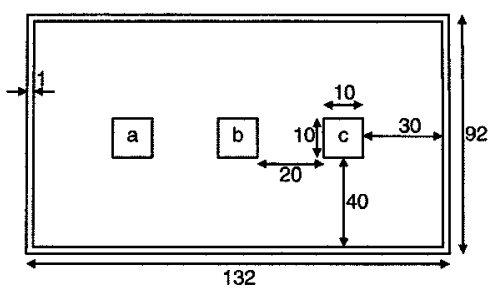

Fig. 4. Enclosed three-phase bus-bar problem (dimensions in $\mathrm{mm}$ ).

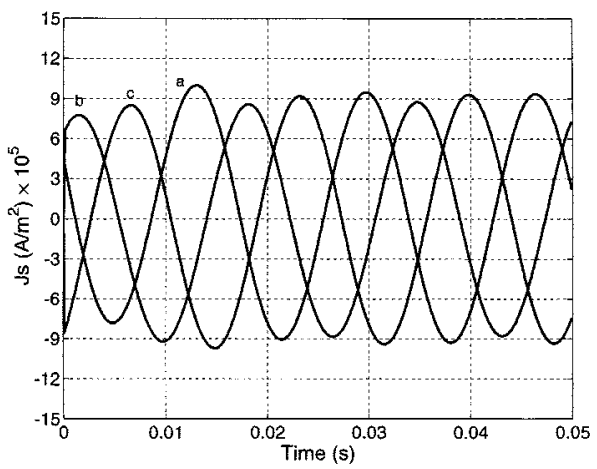

Fig. 5. Bus-bar problem: SCDs at the center of each conductor obtained with Formulation I.

2, 3 (numbered left to right), respectively, for four cases. Interestingly, Formulation I yields better results than Formulation II. Note how the errors increase with excitation frequency and conductor dimensions. Since Formulation I ignores the dependence of total current density on position and simplifies the integration in (3), the error increases with the cross-sectional area of the source conductor. Furthermore, Formulation II ignores the second term on the right side of (12). The error increases not only with source conductor dimension but also with the time derivative (or $j \omega$ in frequency domain) of the MVP. Section III shows the validity of results obtained with Formulation III; they are used as a basis for comparison instead of exact values.

\section{A Three-Phase Bus-Bar Example}

Fig. 4 shows an enclosed, air-filled, three-phase bus-bar arrangement. Enclosure and bus bars are made of copper. The currents in the three conductors are given by

$$
\begin{aligned}
& i_{a}(t)=I_{o} \cos \left(\omega_{0} t\right)-I_{o} e^{-t / \tau} \\
& i_{b}(t)=I_{o} \cos \left(\omega_{0} t-2 \pi / 3\right)-I_{o} \cos (2 \pi / 3) e^{-t / \tau} \\
& i_{c}(t)=I_{o} \cos \left(\omega_{0} t+2 \pi / 3\right)-I_{o} \cos (2 \pi / 3) e^{-t / \tau}
\end{aligned}
$$

where $I_{o}=100 \mathrm{~A}, \omega_{0}=377 \mathrm{rad} / \mathrm{s}$, and $\tau=0.01 \mathrm{~ms}$. The initial condition is $\left.A_{z}\right|_{t=0}=0$. 


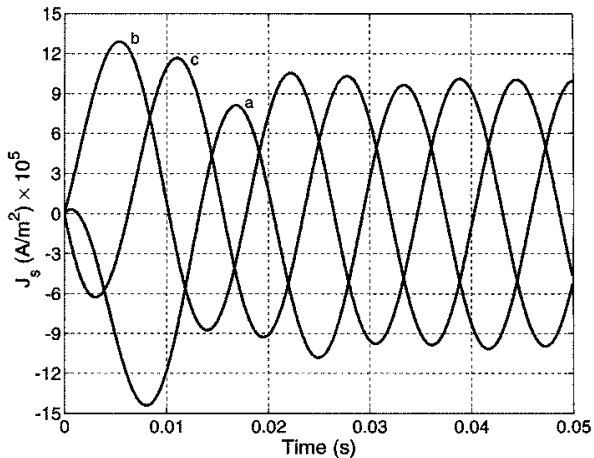

Fig. 6. Bus-bar problem: SCDs obtained with Formulation II.

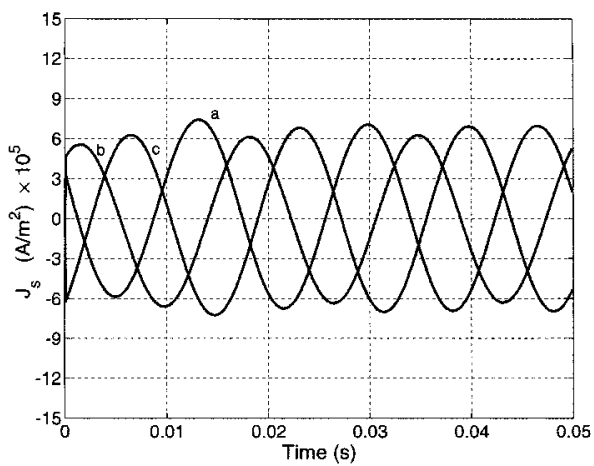

Fig. 7. Bus-bar problem: SCDs obtained with Formulation III.

Figs. 5-7 show the SCDs for each Formulation. Fig. 7 depicts the SCDs computed from the complete equation (12). Comparison of Figs. 6 and 7 shows that the transient behaviors are different. Also, the effects of a nonsymmetrical configuration are manifested in Fig. 7 but not in Fig. 6. Figs. 5 and 7 show similar behavior but different SCD magnitudes.

\section{CONCLUSION}

Three FE formulations for 2-D eddy current and skin effect problems are compared by using two different examples. A 1-D test problem is used to validate Formulation III, which is extended also to frequency domain. Formulation III completely encompasses proximity and skin effects whereas the two earlier formulations do not. Maxwell's equations are not fully applied when the incomplete equation for SCD is invoked. The error resulting from the use of the incomplete equations for current densities increases with frequency and conductor dimensions. The results also indicate that the error for Formulation I is less than the error for Formulation II.

\section{APPENDIX}

Consider the Maxwell's equations for eddy current and skin effect problems:

$$
\begin{aligned}
& \operatorname{curl} \vec{E}=-\frac{\partial \vec{B}}{\partial t} \\
& \operatorname{curl} \vec{H}=\vec{J}
\end{aligned}
$$

where $\vec{B}$ is magnetic flux density, $\vec{H}$ is magnetic field intensity, $\vec{E}$ is electric field intensity, and $\vec{J}$ is TCD. The relevant constitutive relationships are $\vec{B}=\mu \vec{H}$ and $\vec{J}=\sigma \vec{E}$. The MVP is defined by $\vec{B}=$ curl $\vec{A}$. For 2-D analysis, $\vec{A}=A_{z} \vec{a}_{z}$ is assumed. For the $k$ th conductor, (26) and (27) become

$$
\begin{aligned}
& E_{z k}=E_{s k}-\frac{\partial A_{z}}{\partial t} \\
& \operatorname{curl} \frac{1}{\mu} \operatorname{curl} A_{z}=J_{z k} .
\end{aligned}
$$

Substitution of (28) into $J_{z k}=\sigma E_{z k}$ yields

$$
J_{z k}=J_{s k}-\sigma \frac{\partial A_{z}}{\partial t}
$$

where $J_{s k}=\sigma E_{s k}$ is an unknown constant. Since $\operatorname{div} A_{z}=0$, (29) becomes $\operatorname{div}(1 / \mu) \operatorname{grad} A_{z}=-J_{z k}$. Integrate both sides of (27) over the $k$ th conductor cross-section to obtain

$$
\iint_{R_{k}}(\operatorname{curl} \vec{H}) \cdot \overrightarrow{d s}=\iint_{R_{k}} \vec{J}_{z k} \cdot \overrightarrow{d s}=i_{k}(t)
$$

By substituting (30) into (31) and noting that $J_{s k}$ is constant, one obtains

$$
J_{s k}=\frac{i_{k}(t)}{S_{k}}+\frac{\sigma}{S_{k}} \frac{\partial}{\partial t} \iint_{R_{k}} A_{z} d s
$$

\section{REFERENCES}

[1] B. Il Kwon, S. J. Park, and S. C. Park, "Forward, converter analysis by the method of coupling electromagnetic field with hysteresis and circuit equation," IEEE Trans. Magn., vol. 36, pp. 1426-1430, July 2000.

[2] M. C. Costa, S. I. Nabeta, and J. R. Cardoso, "Modified nodal analysis applied to electric circuits coupled with FEM in the simulation of a universal motor," IEEE Trans. Magn., vol. 36, pp. 1431-1434, July 2000.

[3] R. Ludwig and X.-W. Dai, "Numerical and analytical modeling of pulsed eddy currents in a conducting half-space," IEEE Trans. Magn., vol. 26, pp. 299-307, Jan. 1990.

[4] Z. W. Shi and C. B. Rajanathan, "Computation of nonlinear transient eddy current electromagnetic fields in devices driven by power electronic circuits," IEEE Trans. Magn., vol. 33, pp. 1690-1693, Mar. 1997.

[5] P. P. Silvester and R. L. Ferrari, Finite Elements for Electrical Engineers. Cambridge, UK: Cambridge University Press, 1983.

[6] M. V. K. Chari and S. J. Salon, Numerical Methods in Electromagnetism. San Diego, CA: Academic, 2000.

[7] I. A. Tsukerman, A. Konrad, and J. D. Lavers, "A method for circuit connections in time-dependent eddy current problems," IEEE Trans. Magn., vol. 28, pp. 1299-1302, Mar. 1992.

[8] V. K. Garg and J. Weiss, "Finite element solution of transient eddycurrent problems in multiply-excited magnetic systems," IEEE Trans. Magn., vol. MAG-22, pp. 1257-1259, Sept. 1986.

[9] A. Konrad, "Finite element formulation of two-dimensional steady-state skin effect problems: An integrodifferential approach," IEEE Trans. Magn., vol. MAG-18, pp. 284-292, Jan. 1982.

[10] R. Jafari Shapoorabadi, A. N. Sinclair, and A. Konrad, "Finite element determination of the absolute magnitude of an ultrasonic pulse produced by an EMAT," in Proc. 2000 IEEE Int. Ultrasonics Symp., vol. I, San Juan, PR, Oct. 22-25, 2000, pp. 737-741.

[11] R. Jafari Shapoorabadi, A. Konrad, and A. N. Sinclair, "Improved finite element method for EMAT analysis and design," in 8th Joint MMMIntermag Conf., San Antonio, TX, Jan. 7-11, 2001.

[12] - "Improved finite element method for EMAT analysis and design," IEEE Trans. Magn., vol. 37, pp. 2821-2823, July 2001. 